\title{
THE MOST GENERAL CLOSED POINT SET OVER WHICH CONTINUOUS FUNCTION MAY BE DEFINED BY CERTAIN PROPERTIES*
}

BY G. T. WHYBURN

In a recent paper $\dagger \mathrm{C}$. $\mathrm{H}$. Rowe states the following theorem.

THEOREM A. In order that a function $f(x, y, \cdots)$, defined in a finite open region $R$, may be continuous in $R$, it is necessary and sufficient that, as we move along any continuous curve whose points belong to $R$, the function should never pass from one value to another without taking every intermediate value, and that, for every value of $\alpha$, the set $E$ of all points of $R$ for which $f=\alpha$ should contain all those of its limit points that belong to $R$.

Rowe points out difficulties in extending his theorem to functions defined over a closed region $\ddagger$ instead of an open one. $\mathrm{He}$ states that his theorem is valid for functions defined over a closed region $R$ provided $R$ satisfies the condition that for every one of its boundary points $P$ there are values of $\delta$ as small as we please such that the points of $R$ whose distances from $P$ do not exceed $\delta$ form a set having the property that every two of its points can be joined by an arc of a continuous curve which lies wholly in this set. The present paper has two main purposes: (1) to show that the condition imposed by Rowe upon the closed region $R$ above is unnecessarily strong and (2) to give a condition on a closed point set $M$, whether $M$ is a closed region or not, which is both necessary and sufficient in order that Theorem A should be valid for functions defined over $M$.

* Presented to the Society, September 9, 1926.

$\dagger$ Note on a pair of properties which characterize continuous functions, this Bulletin, vol. 32 (1926), pp. 285-287. loc. cit.

$\ddagger$ For definitions of the terms open region and closed region see Rowe, 
In view of Theorem I below, the following example of a simple closed curve plus its interior which does not satisfy Rowe's condition shows that his condition is much too strong. Let $t_{1}$ denote the simple continuous arc composed of the point $(0,0)$ together with the broken line joining, in the order here named, the points $(4,0),(4,1),(-4,1)$, $(-4,-1),(2,-1),\left(2, \frac{1}{2}\right),\left(-2, \frac{1}{2}\right),\left(-2,-\frac{1}{2}\right),\left(1,-\frac{1}{2}\right)$, $\left(1, \frac{1}{4}\right),\left(-1, \frac{1}{4}\right),\left(-1,-\frac{1}{4}\right), \cdots$, indefinitely. Let $t_{2}$ denote the simple continuous arc consisting of the point $(0,0)$ plus the broken line joining, in the order here named, the points $(3,0),\left(3, \frac{3}{4}\right),\left(-3, \frac{3}{4}\right),\left(-3,-\frac{3}{4}\right),\left(\frac{3}{2},-\frac{3}{4}\right),\left(\frac{3}{2}, \frac{3}{8}\right),\left(-\frac{3}{2}, \frac{3}{8}\right)$, $\left(-\frac{3}{2},-\frac{3}{8}\right),\left(\frac{3}{4},-\frac{3}{8}\right),\left(\frac{3}{4}, \frac{3}{16}\right), \cdots$, indefinitely. Let $I$ denote the straight line interval from $(3,0)$ to $(4,0)$. It is easy to see that if $J$ denotes the point set $t_{1}+t_{2}+I$, then $J$ is a simple closed curve. Let $R$ denote $J$ plus its interior. Then $R$ is a closed region. But if $P$ denotes the point $(0,0)$, then no matter what positive number $\delta$ we take which is less than 1 , the set $K_{\delta}$ of all those points of $R$ whose distances from $P$ do not exceed $\delta$ is not connected. Hence $R$ does not satisfy Rowe's condition, even though it belongs to one of the simplest types of closed regions.

THEOREM I. In order that Theorem A should be valid for functions defined over a closed point set $M$ it is necessary and sufficient that $M$ should be connected im kleinen.*

Proof. The condition is sufficient. Let $f(x, y, \cdots)$ be any functon which is defined over a closed and connected im kleinen point set $M$ and which satisfies the conditions of Theorem A. Let $P$ denote any definite point of $M$, and $\epsilon$ any positive number. Let $E_{1}$ and $E_{2}$ respectively denote the

* A point set $M$ is connected im kleinen provided that for each point $P$ of $M$ and for every positive number $\epsilon$ there exists a positive number $\delta_{\epsilon} P$ such that every point $X$ of $M$ whose distance from $P$ is less than $\delta_{\epsilon} P$ lies, together with $P$, in a connected subset of $M$ of diameter less than $\epsilon$. By the diameter of a point set $K$ is meant the upper limit of the aggregate of numbers $\delta(X, Y)$, where $X$ and $Y$ are any pair of points of $K$ and $\delta(X, Y)$ is the distance between $X$ and $Y$. The notation $\delta(X, Y)$, to represent the distance between the points or point sets $X$ and $Y$, will be used in this paper. See Hahn, Mengentheoretische Charakterisierung der stetigen Kurve, Wiener Akademie Sitzungsberichte, vol. 123, pp. 2433-2489. 
set of all those points $X$ of $M$ such that $f(X)=f(P)+\epsilon$ and $f(X)=f(P)-\epsilon$, respectively. By hypothesis both $E_{1}$ and $E_{2}$ are closed or vacuous point sets, and neither contains the point $P$. Hence, there exists a positive number $e$ such that no point of $M$ whose distance from $P$ is $\leqq e$ belongs to either $E_{1}$ or $E_{2}$. Since $M$ is connected im kleinen and closed, there exists a positive number $d$ such that every point $Q$ of $M$ such that $\delta(Q, P)<d$ can be joined to $P$ by a simple continuous arc* every point of which belongs to $M$ and is at a distance less than $e$ from $P$. Let $K$ denote the set of all those points of $M$ whose distance from $P$ is less than $d$. Then for every point $Q$ of $K, f(P)-\epsilon<f(Q)<f(P)+\epsilon$. For if for some point $A$ of $K$, this statement is not true, then since $A$ can be joined to $P$ by an arc $t$ which lies in $M$ but which has no point in common with either of the sets $E_{1}, E_{2}, f$ does not take on all values between $f(A)$ and $f(P)$ as we move along $t$ from $A$ to $P$, contrary to hypothesis. Hence, $f(x, y, \cdots)$ is continuous at every point $P$ of $M$.

The condition is also necessary. Let $M$ denote any closed point set which is not connected im kleinen. I will show that a function can be defined over $M$ which will satisfy the conditions of Theorem A but which will not be continuous at every point of $M$. Since $M$ is not connected im kleinen, there exists a point $P$ of $M$ and a positive number $\epsilon$ such that no matter what positive number $\delta$ may be, there exists some point $Q$ of $M$ such that $\delta(Q, P)<\delta$ but such that $Q$ and $P$ do not lie together in any connected subset of $M$ of diameter less than $\epsilon$. It easily follows that $M$ contains a sequence of points $P_{1}, P_{2}, P_{3}, \cdots$, having $P$ as its sequential limit point and such that for no positive integer $n$ does $P_{n}$ lie together with $P$ in a connected subset of $M$ of diameter $\leqq \epsilon / 2$. Let $K$ denote the set of all those points of

* Sec R. L. Moore, A theorem concerning continuous curves, this Bulletin, vol. 23 (1917), pp. 233-236; J. R. Kline, Concerning approachability of simple closed and open curves, Transactions of this Society, vol. 21 (1920), footnote to p. 453, and S. Mazurkiewicz, Sur les lignes de Jordan, Fundamenta Mathematicae, vol. 1 (1920), pp. 166-209. 
$M$ which can be joined to $P$ by a connected subset of $M$ of diameter $\leqq \epsilon / 2$. Since $M$ is closed, it easily follows that $K$ is closed. Let $C$ denote the set of all points whose distances from $P$ are equal to $\epsilon / 2$. Now let us define a function $f(x, y, \cdots)$ over $M$ as follows. For every point $X$ of $M$ which is on $K$ let $f(X)=\delta(X, C)$, and for every point $X$ of $M$ which is not on $K$ let $f(X)=\delta(X, K)$.

It is easy to see that $f(x, y, \cdots)$, thus defined over $M$ satisfies both conditions of Theorem A. But since no point of the sequence $P_{1}, P_{2}, P_{3}, \cdots$, belongs to $K$, and since $\delta\left(P_{n}, P\right) \rightarrow 0$ as $n \rightarrow \infty$, then $f\left(P_{n}\right) \rightarrow 0$ as $n \rightarrow \infty$. But $f(P) \neq 0$. Hence $f(x, y, \cdots)$ is not continuous at $P$. It follows that the condition of Theorem I is necessary.

TheOREM II. In order that Theorem $A$ should be valid for functions defined over a point set $M$, (closed or not), it is sufficient that $M$ should be arcwise connected im kleinen.*

Theorem II can be proved by an argument almost identical with the first part of the proof of Theorem I.

It is interesting to note that Theorem A is a special case of Theorem II, since every open region is arcwise connected im kleinen.

It can easily be shown by means of an example similar to the one given in the second part of the proof of Theorem I that in order that Theorem A should be valid for functions defined over a point set $M$, (closed or not), it is necessary that $M$ should be connected im kleinen. It would be interesting to determine a necessary and sufficient condition on a point set $M$, which is otherwise unrestricted, in order that Theorem A should be valid for functions defined over $M$.

The University of Texas

* A point set $M$ is arcwise connected im kleinen provided that for every point $P$ of $M$ and every positive number $\epsilon$ there exists a positive number $\delta \epsilon_{P}$ such that every point $X$ of $M$ such that $\delta(X, P)<\delta_{\epsilon} P$ can be joined to $P$ by a simple continuous arc which is a subset of $M$ and is of diameter less than $\epsilon$. The properties "connected im kleinen" and "arcwise connected im kleinen" are equivalent for closed point sets. 